\title{
TS-GOEA: a web tool for tissue-specific gene set enrichment analysis based on gene ontology
}

\author{
Jiajie Peng ${ }^{1}$, Guilin Lu ${ }^{1}$, Hansheng Xue ${ }^{1}$, Tao Wang ${ }^{2}$ and Xuequn Shang ${ }^{1 *}$ \\ From Biological Ontologies and Knowledge bases workshop at IEEE BIBM (2018) \\ Madrid, Spain 3-6 December 2018
}

\begin{abstract}
Background: The Gene Ontology (GO) knowledgebase is the world's largest source of information on the functions of genes. Since the beginning of GO project, various tools have been developed to perform GO enrichment analysis experiments. GO enrichment analysis has become a commonly used method of gene function analysis. Existing GO enrichment analysis tools do not consider tissue-specific information, although this information is very important to current research.

Results: In this paper, we built an easy-to-use web tool called TS - GOEA that allows users to easily perform experiments based on tissue-specific GO enrichment analysis. TS - GOEA uses strict threshold statistical method for $\mathrm{GO}$ enrichment analysis, and provides statistical tests to improve the reliability of the analysis results. Meanwhile, TS - GOEA provides tools to compare different experimental results, which is convenient for users to compare the experimental results. To evaluate its performance, we tested the genes associated with platelet disease with TS - GOEA.

Conclusions: TS - GOEA is an effective GO analysis tool with unique features. The experimental results show that our method has better performance and provides a useful supplement for the existing GO enrichment analysis tools. TS - GOEA is available at http://120.77.47.2:5678.
\end{abstract}

Keywords: Gene ontology, Enrichment analysis, Tissue-specific, Web tool

\section{Background}

The Gene Ontology (GO) knowledgebase is the world's largest source of information on the functions of genes. This knowledge is both human-readable and machinereadable, and is a foundation for computational analysis of large-scale molecular biology and genetics experiments in biomedical research. The goal of the Gene Ontology Consortium is to produce a dynamic, structured, controlled vocabulary that cover several domains of molecular and cellular biology [1]. GO and GO annotations provide a convenient way for biologists to explore the function of gene sets in biological experiments. In detail,

${ }^{*}$ Correspondence: shang@nwpu.edu.cn

'School of Computer Science, Northwestern Polytechnical University, 710129 Xi'an, China

Full list of author information is available at the end of the article
GO terms represent a kind of biological knowledge which describes the functions of genes and corresponding gene products [2]. As a unified knowledge base, GO provides three accessible independent ontology, namely biological processes(BP), cellular components(CC) and molecular functions(MF). GO has been widely used in molecular biology and genomics research to describe gene products $[1,3]$. In addition, $\mathrm{GO}$ provides an ontology annotation system that associates genes or gene products with GO terminology to form a "snapshot" of current biological knowledge. Biologists can design experiments based on GO to verify their biological hypothesis $[1,3-8]$.

Gene function inference is important in lots of researches [9-12]. The goal of Gene Ontology Enrichment Analysis (GOEA) is to use the annotations of the gene set to find out which GO terms are overrepresented or 
underrepresented [13, 14]. GOEA has become a common method for functional research of large-scale genome or transcriptome data [15]. Existing GOEA tools can be summarized into two categories, web-based and offlinebased application. Offline-based tools require users to download the package and install a local environment, such as BinGO [16], which is not convenient for users to use. At the same time, web-based GOEA tools are very popular with biologists because of its simplicity and convenience, such as DAVID [17], g:profiler [18], GOEAST [15] and GOrilla [19]. However, current GOEA tools do not consider tissue-specific information, and most existing biological experiments do not focus on tissue-specific gene regulation, ignoring their importance in their respective networks [20,21]. Although all human tissues have a common process, the gene expression patterns of tissues are different, which means that different regulatory procedures control the specificity of the tissue, gene regulation is understood differently in different tissues [21]. Understanding the specific expression and regulation of genes in different tissues is helpful to better understand the genetic relationship and etiology of tissues, as well as to discover new tissue-specific drug targets [22]. Therefore, it is very important to consider tissue-specific genes in current research.

In addition, existing tools simply show the results of enrichment analysis, but they do not show users the relationship between those GO Terms in the results of enrichment analysis. We believe that visualizing the relationship between these GO terms can help us better understand our experimental results.

In order to improve these shortcomings mentioned above, based on Homo sapiens' GO Annotated data and The Genotype-Tissue Expression [23] data, we constructed an easy-to-use web tool called TS - GOEA, which allows users to easily conduct experiments based on organization-specific Go enrichment analysis. It uses appropriate statistical methods to determine whether the Go term significantly enriches specific organizations based on a given gene list. Compared with existing tools, it has the following advantages:

- As far as we know, TS - GOEA is the first tool to provide GO enrichment analysis based on Tissue specificity.

- TS - GOEA is an easy-to-use Web application that provides an intuitive visual interface that shows the location of specific GO terms in the ontology, as well as the relationships between all enriched Go terms.

- $T S-G O E A$ can save the results of many experiments, and support the comparison between the results of two groups of different experiments.

\section{Materials and methods}

$T S-G O E A$ is a Web tool with three main layers: data support layer (back-end annotation database); data mining layer (algorithm and statistics); and result presentation layer (interface). The whole framework of $T S-G O E A$ is shown in Fig. 1 and the workflow of TS - GOEA is shown in Fig. 2.

\section{Data resource}

The data used by TS - GOEA comes from the following resources. The GO ontology file is downloaded from the Gene Ontology Project website(http://www. geneontology.org/). All GO term definitions and hierarchical relationships are extracted from the ontology file. The GO annotation file is downloaded and parsed from the Gene Ontology Project website to extract relevant

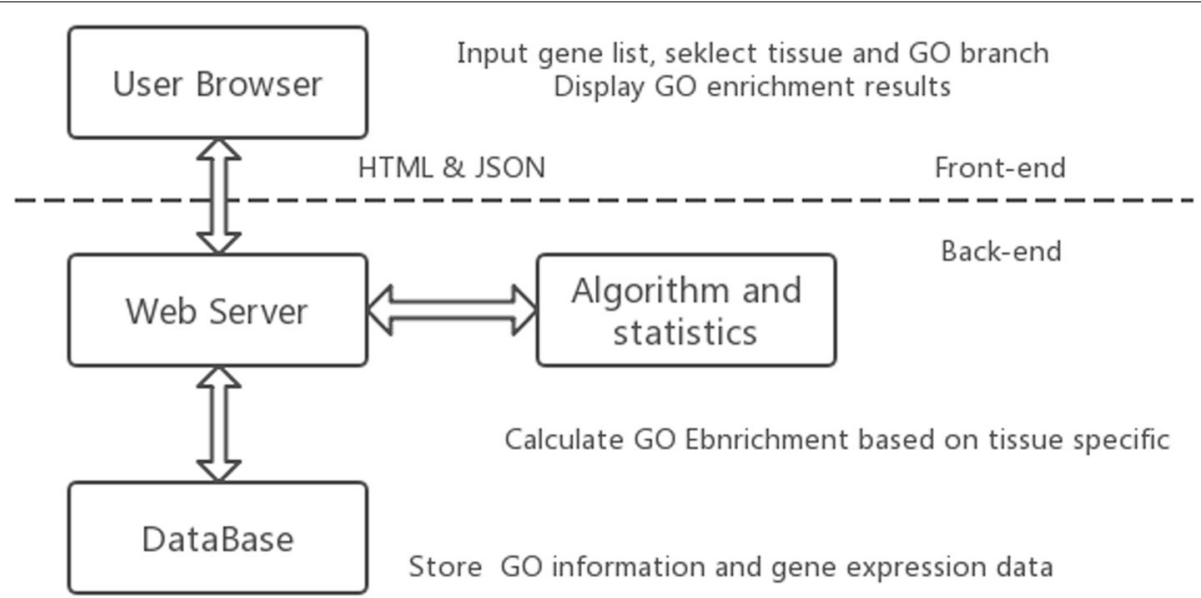

Fig. 1 The whole framework of TS-GOEA. The front-end provides a user browser which inputs gene list and displays corresponding GO enrichment results. Calculating $\mathrm{GO}$ enrichment based on Tissue specific is finished in the back end of TS - GOEA 


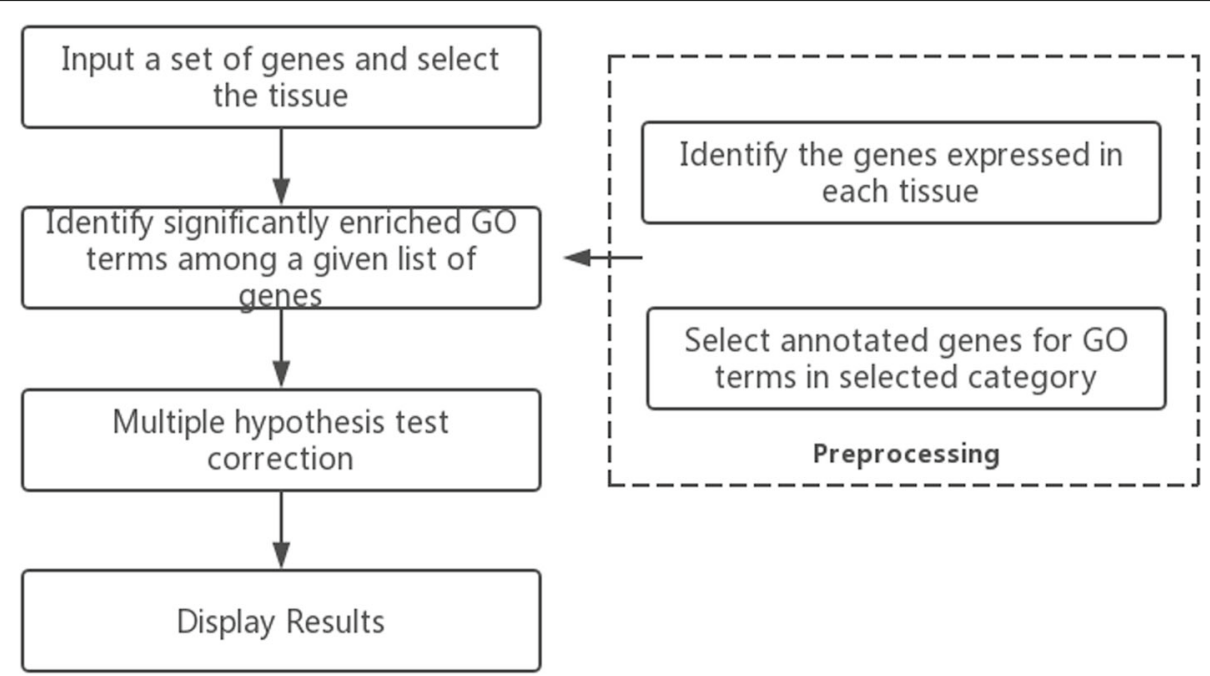

Fig. 2 Workflow of tissue-specific GO enrichment analysis

GO terms. Gene expression data was downloaded from the GTEx website(https://gtexportal.org/) and genes for tissue-specific expression were calculated.

\section{Inputs and outputs format}

$T S-G O E A$ requires the user to enter a list of genes, we currently use as UniPortKB identifier. Besides, TS-GOEA provides three types of output files:

- HTML table, which describes detailed information of enriched GO terms and corresponding NCBI links.

- Plain text files of GO terms for local processing and analysis.

- Graphical Visualization, showing the hierarchical relationships between all enriched GO Term in the GO category and the hierarchical relationships of each GO term.

\section{Identify genes expressed on different tissues}

Strictly, tissue-specific genes refer to genes whose function and expression are limited to specific tissue or cell types. In many cases, however, the concept of specificity has been extended to tissue selectivity, where gene expression is abundant in one or more tissue/cell types.

The Genotype Tissue Expressions (GTEX) project aims to establish a common resource database and related organization library for studying the relationship between genetic variation and gene expression and other molecular phenotypes in a variety of reference tissues [23, 24]. For ease of study, GTEx dataset provides Transcripts per Million (TPM) value and read counts of genes in different tissues. Select genes that are specifically expressed in tissues based on the following principles [25]:
- In at least $20 \%$ samples, TPMs fraction is greater than or equal to 0.1 .

- in at least $20 \%$ of samples, reads (unnormalized) greater than or equal to 6

\section{Hypergeometric test}

TS - GOEA uses hypergeometric testing to calculate possibility. The $p$-value could be calculated as:

$$
P(X=x>k)=\sum_{x=k}^{M} \frac{\left(\begin{array}{c}
M \\
x
\end{array}\right)\left(\begin{array}{c}
N-M \\
n-x
\end{array}\right)}{\left(\begin{array}{l}
N \\
n
\end{array}\right)}
$$

Where, $N$ is the size of genes specifically expressed in the tissue selected, for a given GO term, there are $M$ genes within $N$ associated with it, and $n$ is the size of genes in the input gene list, $k$ is the size of the genes of interaction between $n$ and $M$ [15]. TS-GOEA use the Benjamini Hochberg method to adjust the original $\mathrm{p}$ value to the error detection rate (FDR) to avoid multiple test problems that may lead to excessive false positive results [26].

\section{Features of TS - GOEA}

The primary function of TS - GOEA is to identify statistically enriched GO terms in a given list of genes. As a web-based GO enrichment analysis tool, TS - GOEA has the following improvements or unique features compared to available tools.

\section{Tissue specificity}

None of the current GO enrichment analysis tools can take into tissue-specific information account. However, studying the tissue-specific genes is an important step in 
understanding the progress of life activities and organizational functions. TS - GOEA performs GO enrichment analysis based on tissue specific information can effectively supplement the shortcomings of current research and better explain the results of biological experiments.

\section{Graphical visualization}

The GO terms in each ontology category are not independent but are located in the same branch, with a hierarchical relationship to each other. Understanding the locational relationships of GO terms may help users better understand their results. For Example, the relative position relationship of GO:0001228 in gene ontology is shown in Fig. 3. With the GO lineage diagram, one can easily understand the enriched GO terms and its hierarchical relationship in GO.

\section{Multiple experiments comparison function}

A unique feature of $T S-G O E A$ is to allow comparison of GO terminology enrichment states for different experimental results. Users can upload the results of the GO enrichment analysis provided by TS - GOEA to the website, or add the results to the comparison page, and compare the similarities and differences between the two experimental results using the Venn diagram.

\section{Highly interactive}

The application is highly interactive and can generate different diagrams according to user's selection. For example, in the input interface, users can freely choose interested tissue and a GO category. In the output interface, users can easily download or display their own result. In the result display interface, users can click the GO term list and gene list in the results to view detail information. Users can also compare the results of two enrichment results by adding their job IDs.

We will compare the results of two different GO enrichment analysis at the end of the article to show the advantage of tissue-specific GO enrichment analysis.

\section{Results and discussion}

In this case, tissue-specific genes are defined as a group of genes that express in one or several tissues. Identification of these genes contributes to a better understanding of tissue genetic relationships and pathogenicity [22]. However, due to the complex clinical characteristics and

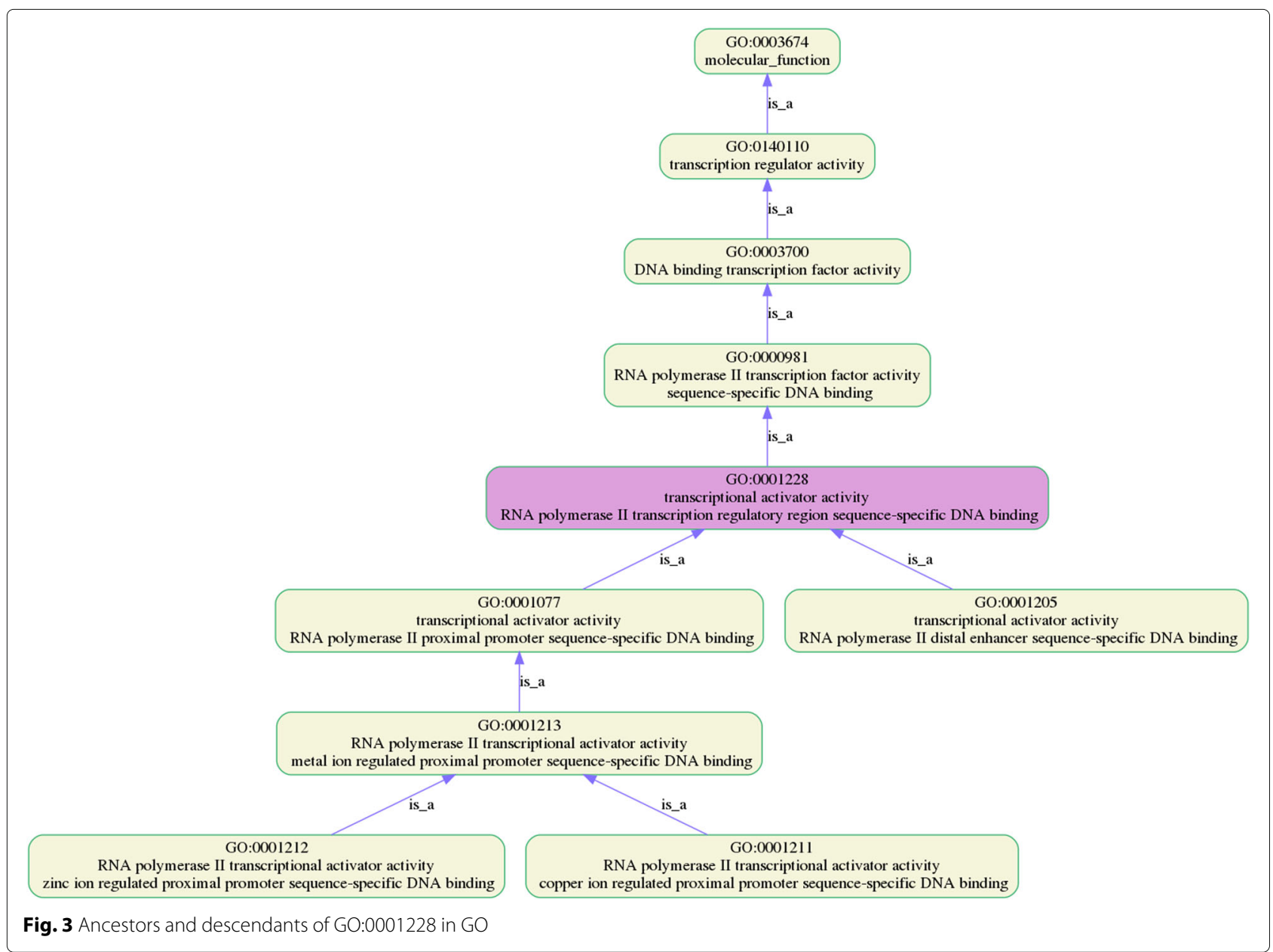




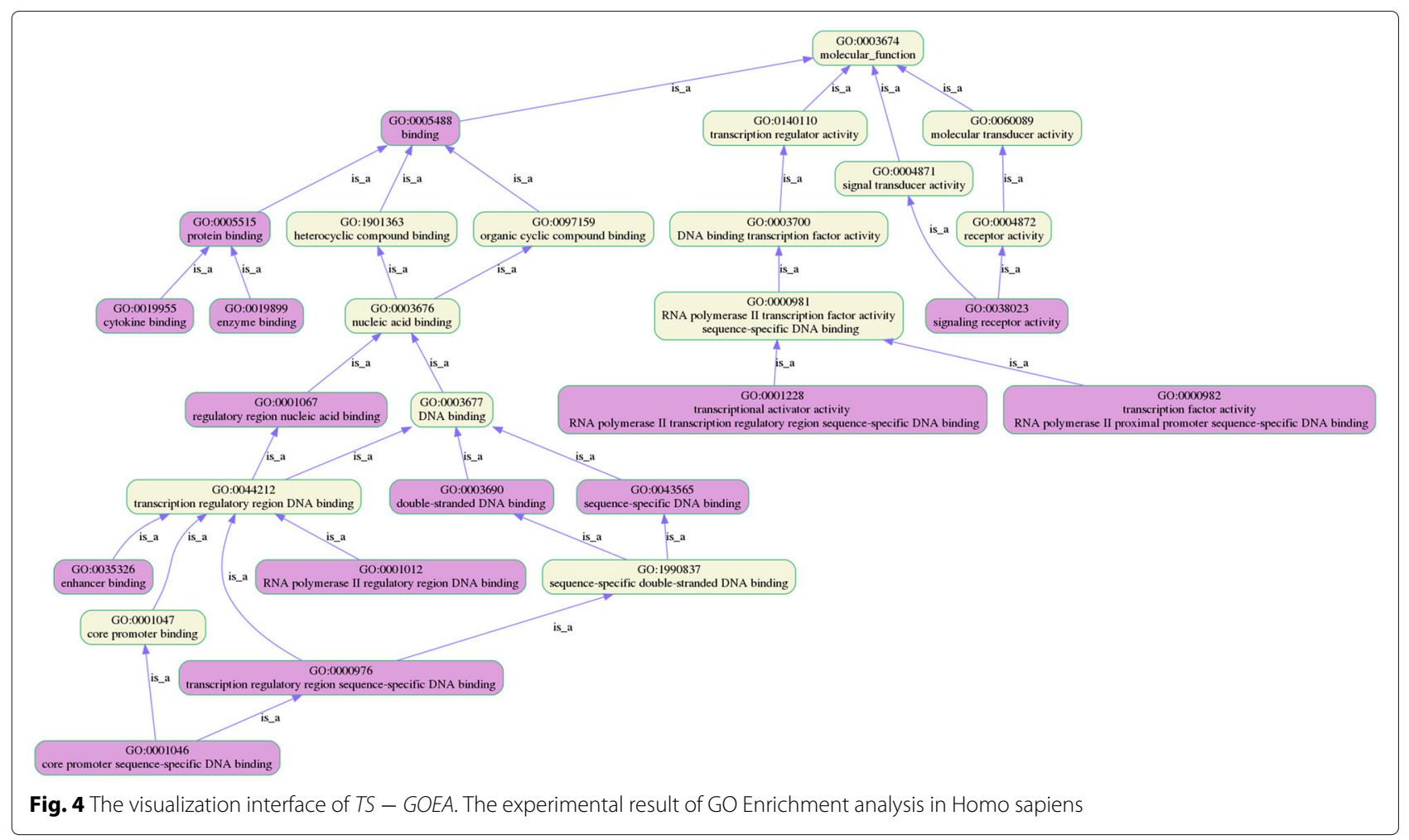

highly heterogeneous genetic background of some diseases, it is difficult to make accurate diagnosis $[27,28]$. It is of great significance to reveal the molecular mechanism of biomedicine by using disease genome to performing the tissue-specific GO enrichment analysis, and then continue to excavate the results and analyze the biological process or signal pathway in which genes may be involved.
Platelet disease is a hemorrhagic disease caused by a defect in the quantity or quality of platelets. It is not difficult to understand that compared to other organizations. In the process of exploring the pathogenesis of disease, the study of blood tissue can obtain more accurate results. Therefore, we performed GO enrichment analysis in blood tissue to verify the performance of our tools.

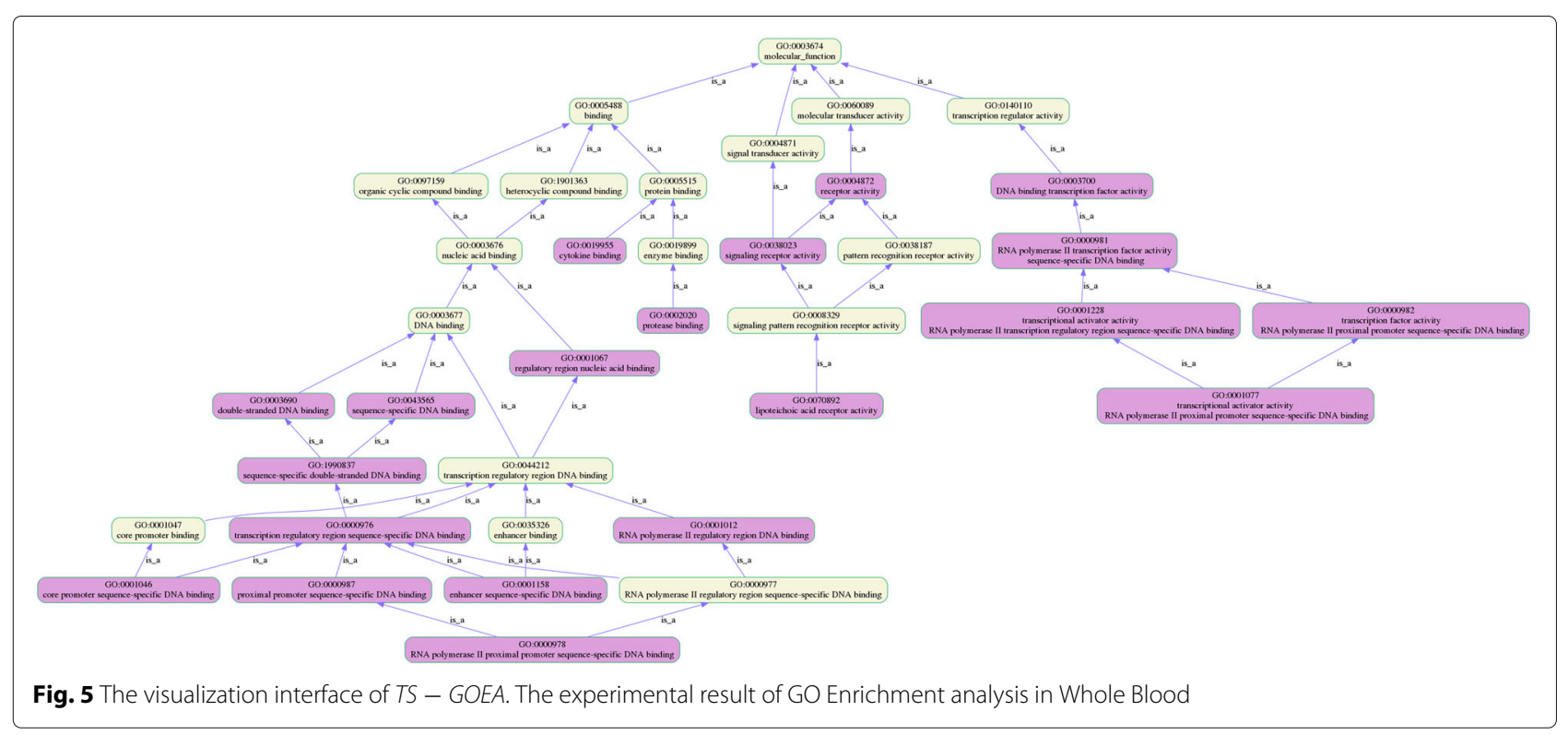




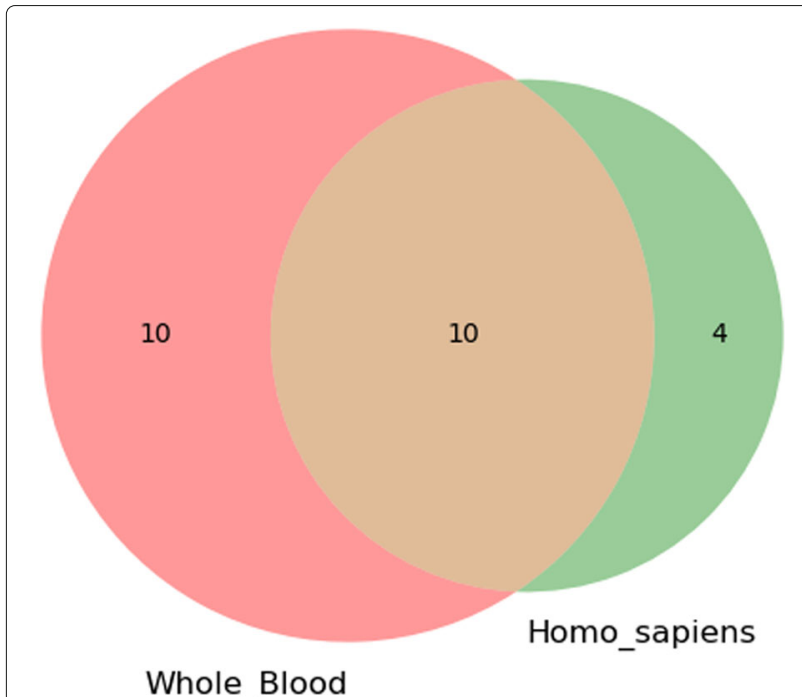

Fig. 6 The Venn diagram of two groups of results, which was implemented through the pairwise comparison tool of TS - GOEA

To test TS-GOEA, we performed GO enrichment analysis in whole blood tissues and identified a set of GO terms for genes associated with platelet disease. Then, we carried out GO enrichment analysis without using tissuespecific information, and obtained another set of data. We compared and explained the differences between the two groups of experiments. Using the structural relationship of GO, we plot GO lineage images with the results of the two groups of enrichment analysis. Figure 4 shows the result of GO enrichment analysis in Homo sapiens, and Fig. 5 shows the result of GO enrichment analysis in Whole Blood. We compare the two groups of results, as shown in Fig. 6.

By comparing the results of the two sets of experiments, we can find that the GO enrichment analysis based on whole blood tissue produces more accurate and effective results. More specifically, in order to facilitate comparison, we list the GO terms for these differences in the Table 1. Most of these GO terms listed are related to the activity of proteases and DNA binding processes, and are helpful to mediate the transcription process. We enumerate the genes annotated by those GO Terms and search these genes on eDGAR [29]. We found that these genes affect the formation and function of related proteases in the blood, and their abnormal expression can lead to some blood-related diseases, including Platelet disease, which proves the effectiveness of our tools. The results showed that tissue-specific GO enrichment analysis could show information at a more specific level. Therefore, we believe that our tools can help biologists complement and improve the process of biological experiments, understand their results from a functional point of view, and explore the potential molecular mechanisms behind biological processes [15].

\section{Conclusion}

Since the beginning of the GO project, GO enrichment analysis has become a widely used method in the functional study of large-scale genome or transcriptome data. Various tools have been developed to support the exploration and search of the go database. Specifically, various tools are currently available to perform GO enrichment analysis. However, existing tools ignore tissue-specific information, which may bias the results of biological experiments.

In this article, we developed a Web application that allows users to perform organization-specific GO rich analysis experiments, and we also visualize our results to facilitate users to view the relationships between GO terms. We also provide tools to compare different experimental results, so users can find similarities and differences between different experiments and mine deeper relationships. In a word, TS-GOEA is an easy-to-use Web

Table 1 GO TERMS ENRICHED IN WHOLE BLOOD and related disease genes

\begin{tabular}{lll}
\hline GO term & GO function & Disease-related gene \\
\hline G0:0000978 & RNA polymerase II proximal promoter sequence-specific DNA binding & GATA1,HOXD13CEBPA,FLI1,NR4A3,CEBPA \\
G0:0000981 & $\begin{array}{l}\text { RNA polymerase II transcription factor activity, sequence-specific DNA } \\
\text { binding }\end{array}$ & FLI1,CEBPA,GATA1,GATA2,HOXD13,NR4A3 \\
G0:0000987 & proximal promoter sequence-specific DNA binding & CEBPA,GATA1,HOXD13,NR4A3 \\
G0:0001077 & $\begin{array}{l}\text { transcriptional activator activity, RNA polymerase Il proximal promoter } \\
\text { sequence-specific DNA binding }\end{array}$ & GATA1,GATA2,HOXD13,CEBPA,FL11,NR4A3,CEBPA \\
G0:0001158 & enhancer sequence-specific DNA binding & GATA1,GATA2,HOXD13 \\
G0:0002020 & protease binding & WWF,ITGB,ELANE,NR4A3 \\
G0:0003700 & obsolete negative regulation of diuresis & GATA1,GATA2,HOXD13,CEBPA,FL11,CEBPA \\
G0:0004872 & receptor activity & TBXA2R,CD36,F2,GP1BB,MPL,NR4A3 \\
G0:0070892 & lipoteichoic acid receptor activity & CD36 \\
G0:1990837 & sequence-specific double-stranded DNA binding & CEBPA,GATA1,GATA2,HOXD13,NR4A3 \\
\hline
\end{tabular}


application that fills the gap in the field of tissue-specific GO enrichment analysis and can effectively supplement the conclusions of some current biological experiments.

\author{
Abbreviations \\ BP: Biological processes; CC: Cellular components; FDR: False discovery rate; \\ GO: Gene ontology; GOEA: Gene ontology enrichment analysis; GTEx: The \\ genotype-tissue expression; MF: Molecular functions; TPM: Transcripts per \\ Million
}

\section{Acknowledgements}

We are thankful to the reviewers for their helpful comments.

\section{About this supplement}

This article has been published as part of BMC Bioinformatics Volume 20 Supplement 18, 2019: Selected articles from the Biological Ontologies and Knowledge bases workshop 2018. The full contents of the supplement are available online at https://bmcbioinformatics.biomedcentral.com/articles/ supplements/volume-20-supplement-18.

\section{Authors' contributions}

JP and XS designed the web tool framework; JP and GL implemented the web tool; JC, JP and GL wrote this manuscript; HX and TW helped design the visualization interface. All authors read and approved the final manuscript.

\section{Funding}

Publication costs were funded by National Natural Science Foundation of China (No. 61702421, U1811262, 61772426), the international Postdoctoral Fellowship Program (no. 20180029), China Postdoctoral Science Foundation (No. 2017M610651), Fundamental Research Funds for the Central Universities (No. 3102018zy033), Top International University Visiting Program for Outstanding Young scholars of Northwestern Polytechnical University.

\section{Ethics approval and consent to participate}

Not applicable.

\section{Consent for publication}

Not applicable.

\section{Competing interests}

The authors declare that they have no competing interests.

\section{Author details}

${ }^{1}$ School of Computer Science, Northwestern Polytechnical University, 710129 Xi'an, China. ${ }^{2}$ School of Computer Science, Harbin Institute of Technology,

150001 Harbin, China.

Published: 25 November 2019

\section{References}

1. Ashburner M, Ball CA, Blake JA, Botstein D, Butler H, Cherry JM, Davis AP, Dolinski K, Dwight SS, Eppig JT, et al. Gene ontology: tool for the unification of biology. Nature Genet. 2000;25(1):25.

2. Peng J, Li H, Liu Y, Juan L, Jiang Q, Wang Y, Chen J. Intego2: a web tool for measuring and visualizing gene semantic similarities using gene ontology. BMC Genomics. 2016;17(5):553.

3. Consortium GO. The gene ontology (go) database and informatics resource. Nucleic Acids Res. 2004;32(suppl_1):258-61.

4. Consortium GO. Gene ontology consortium: going forward. Nucleic Acids Res. 2014;43(D1):1049-56.

5. Peng J, Guan J, Shang X. Predicting parkinson's disease genes based on node2vec and autoencoder. Front Genet. 2019;10:226.

6. Consortium GO. The gene ontology (go) project in 2006. Nucleic Acids Res. 2006;34(suppl_1):322-6.

7. Peng J, Wang $X$, Shang $X$. Combining gene ontology with deep neural networks to enhance the clustering of single cell rna-seq data. BMC bioinformatics. 2019;20(8):284

8. Peng J, Zhang X, Hui W, Lu J, Li Q, Liu S, Shang X. Improving the measurement of semantic similarity by combining gene ontology and co-functional network: a random walk based approach. BMC Syst Biol. 2018;12(2):18

9. Peng J, Hui W, Li Q, Chen B, Hao J, Jiang Q, Shang X, Wei Z. A learning-based framework for miRNA-disease association identification using neural networks. Bioinformatics. 2019;35(21):4364-71.

10. Cheng L, Wang P, Tian R, Wang S, Guo Q, Luo M, Zhou W, Liu G, Jiang H, Jiang Q. Lncrna2target v2. 0: a comprehensive database for target genes of Incrnas in human and mouse. Nucleic Acids Res. 2018;47(D1):140-4.

11. Peng J, Zhu L, Wang Y, Chen J. Mining relationships among multiple entities in biological networks. IEEE/ACM Trans Comput Biol Bioinforma. 2019. https://doi.org/10.1109/TCBB.2019.2904965.

12. Cheng L, Hu Y, Sun J, Zhou M, Jiang Q. Dincrna: a comprehensive web-based bioinformatics toolkit for exploring disease associations and ncrna function. Bioinformatics. 2018;34(11):1953-6.

13. Ernst J, Bar-Joseph Z. Stem: a tool for the analysis of short time series gene expression data. BMC bioinformatics. 2006;7(1):191.

14. Rodriguez-Esteban R, Jiang X. Differential gene expression in disease: a comparison between high-throughput studies and the literature. BMC Med Genomics. 2017;10(1):59.

15. Zheng Q, Wang X-J. Goeast: a web-based software toolkit for gene ontology enrichment analysis. Nucleic Acids Res. 2008;36(suppl_2):358-63.

16. Maere S, Heymans K, Kuiper M. Bingo: a cytoscape plugin to assess overrepresentation of gene ontology categories in biological networks. Bioinformatics. 2005;21(16):3448-9.

17. Dennis G, Sherman BT, Hosack DA, Yang J, Gao W, Lane HC, Lempicki RA. David: database for annotation, visualization, and integrated discovery. Genome Biol. 2003;4(9):60.

18. Reimand J, Arak T, Vilo J. g: Profiler-a web server for functional interpretation of gene lists (2011 update). Nucleic Acids Res. 2011;39(suppl_2):307-15.

19. Eden E, Navon R, Steinfeld I, Lipson D, Yakhini Z. Gorilla: a tool for discovery and visualization of enriched go terms in ranked gene lists. BMC bioinformatics. 2009;10(1):48.

20. Liu X, Yu X, Zack DJ, Zhu H, Qian J. Tiger: a database for tissue-specific gene expression and regulation. BMC bioinformatics. 2008;9(1):271.

21. Sonawane AR, Platig J, Fagny M, Chen C-Y, Paulson JN, Lopes-Ramos CM, DeMeo DL, Quackenbush J, Glass K, Kuijjer ML. Understanding tissue-specific gene regulation. Cell Rep. 2017;21(4):1077-88.

22. Xiao S-J, Zhang C, Zou Q, Ji Z-L. Tisged: a database for tissue-specific genes. Bioinformatics. 2010;26(9):1273-5.

23. Lonsdale J, Thomas J, Salvatore M, Phillips R, Lo E, Shad S, Hasz R, Walters G, Garcia F, Young N, et al. The genotype-tissue expression (gtex) project. Nature Genet. 2013;45(6):580.

24. Consortium $\mathrm{G}$, et al. The genotype-tissue expression (gtex) pilot analysis: multitissue gene regulation in humans. Science. 2015;348(6235):648-60.

25. Consortium $\mathrm{G}$, et al. Genetic effects on gene expression across human tissues. Nature. 2017;550(7675):204.

26. Benjamini $Y$, Hochberg Y. Controlling the false discovery rate: a practical and powerful approach to multiple testing. J Royal Stat Soc: Ser B (Methodol). 1995;57(1):289-300.

27. Peng J, Hui W, Shang X. Measuring phenotype-phenotype similarity through the interactome. BMC bioinformatics. 2018;19(5):114.

28. Peng J, Xue H, Shao Y, Shang X, Wang Y, Chen J. A novel method to measure the semantic similarity of hpo terms. IJDMB. 2017;17(2):173-88.

29. Babbi G, Martelli PL, Profiti G, Bovo S, Savojardo C, Casadio R. edgar: a database of disease-gene associations with annotated relationships among genes. BMC Genomics. 2017;18(5):554.

\section{Publisher's Note}

Springer Nature remains neutral with regard to jurisdictional claims in published maps and institutional affiliations. 